\title{
Salud mental y apoyo social percibido en escolares de cuarto grado de secundaria de Instituciones Educativas públicas de Lima Metropolitana
}

\author{
Mental health and social support perceived in fourth grade schools of \\ secondary public Educational Institutions of Lima Metropolitana
}

\author{
Rosa E. Huerta Rosales ${ }^{1}$, Renato Santivañez ${ }^{2}$ Jessica Ramos ${ }^{3}$, José Vega ${ }^{4}$, \\ Sandy Pastor ${ }^{5}$ \\ Universidad Nacional Mayor de San Marcos \\ Juan Escudero ${ }^{6}$ \\ Universidad Privada del Norte
}

Recibido: 15 - $10-19$

Aceptado: $17-12-19$

\begin{abstract}
Resumen
El objetivo del estudio fue analizar la relación entre las conductas problemas con el apoyo social percibido, en una muestra de 627 escolares de cuarto de secundaria de Instituciones Educativas públicas, a quienes se les aplicó una ficha de datos, el Inventario de Problemas Conductuales y la Escala multidimensional del apoyo social percibido. Los resultados establecen que existe relación significativa e inversa entre las conductas problemas con el apoyo social percibido. Cuando existe menor nivel de apoyo social, los escolares varones muestran mayores conductas problema de tipo ansiosa y de relación; y las mujeres problemas sociales y de relación. En la dimensión externalización, existe un predominio de dificultades de conducta; en internalización es la somatización la que alcanza mayor porcentaje. La mayoría de los evaluados percibe el apoyo social total en el nivel medio. Existen diferencias significativas en las conductas problemas, son las mujeres las que presentan estas dificultades y mayor diferencia de medias en contraste con los hombres; ellos perciben mayor apoyo social de la familia en comparación con las mujeres quienes perciben mayor apoyo social de los amigos. El apoyo de la familia y los amigos son relevantes como factor protector de la salud mental en los adolescentes.
\end{abstract}

Palabras clave: Salud mental; apoyo social percibido; conductas problema; adolescentes.

\footnotetext{
1 Docente principal, Universidad Nacional Mayor de San Marcos. E-mail: rhuertar@unmsm.edu.pe

2 Docente asociado, Universidad Nacional Mayor de San Marcos. E-mail: rsantivanezo@unmsm.edu.pe

3 Psicóloga, consultora particular. E-mail:jessicaramosreyes@gmail.com

4 Docente asociado, Universidad Nacional Mayor de San Marcos. E-mail: jvegag@unmsm.edu.pe

5 Psicóloga, consultora particular. E-mail:E-mail:sandy_pastor@hotmail.com

6 Docente, Universidad Privada del Norte. E-mail: psicocarlos@hotmail.com
}

(C) Los autores. Este artículo es publicado por la Revista de Investigación en Psicología de la Facultad de Psicología, Universidad Nacional Mayor de San Marcos. Este es un artículo de acceso abierto, distribuido bajo los términos de la licencia Creative Commons Atribucion - No Comercia_Compartir Igual 4.0 Internacional. (http://creativecommons.org/licenses/by-nc-sa/4.0/) que permite el uso no comercial, distribución y reproducción en cualquier medio, siempre que la obra original sea debidamente citada. 


\begin{abstract}
The objective of the study was to analyze the relationship between behavior problems with perceived social support, in a sample of 627 secondary school students from public Educational Institutions, to whom a data sheet, the Inventory of Behavioral Problems and the Multidimensional scale of perceived social support. The results establish that there is a significant and inverse relationship between problem behaviors with perceived social support. When there is a lower level of social support, male schoolchildren show greater problem behaviors of an anxious type and relationship; and women social and relationship problems. In the outsourcing dimension, there is a predominance of behavioral difficulties; in internalization it is somatization that reaches the highest percentage. The majority of those evaluated perceive total social support at the medium level. There are significant differences in problem behaviors, it is women who present these difficulties and greater difference in means in contrast to men; they perceive greater social support from the family compared to women who perceive greater social support from friends. The support of family and friends are relevant as a protective factor for mental health in adolescents.
\end{abstract}

Keywords: Mental health; perceived social support; problem behaviors; adolescents.

La salud mental hoy en día es eje del desarrollo no solo del individuo sino de la nación, es un componente intrínseco de la salud repercutiendo en todos los ámbitos de nuestra vida, integra aspectos biológicos, psicosociales y culturales, todos ellos asociados con las condiciones de vida de las personas. A pesar de esta relevancia observamos, que "en nuestro país se posterga la inclusión de la salud mental en las políticas públicas y en la agenda nacional" (Defensoría del Pueblo, 2008, p.13).

En el caso de los adolescentes el panorama es alarmante, cerca de 13\% de la población la componen adolescentes entre 12 y 18 años (Instituto Nacional de Estadística e Informática [INEI], 2010), encontrándose en el Perú que en 3,379 educandos (entre 13 a 19 años) más del 75\% presentaron estilos de vida no saludables, como consumo de sustancias nocivas, inicio sexual temprano y descuido en su intervención en actividades que propicien su crecimiento personal e insertarse socialmente (De Irala, Corcuera, Osorio y Rivera, 2010). El Instituto Nacional de Salud Mental Honorio Delgado - Hideyo Noguchi - INSM HD-HN (Nota de Prensa, 2012) manifiesta que el 20\% de niños y adolescentes peruanos tienen afectada su salud mental o tiene alguna vulnerabilidad que puede perjudicar su educación; los trastornos más frecuentes son problemas emocionales (depresión o ansiedad) vinculados a conflictos familiares, seguidos por problemas de conducta, de aprendizaje y del desarrollo, distintas formas de maltrato como el abuso físico, emocional, sexual y la negligencia en el cuidado, (Ministerio de Salud [MINSA], 2017). 
La Salud Mental es conceptualizada como un estado dinámico de bienestar que se construye en los diversos contextos en las que está inserta la persona, como el hogar, la escuela, así como en el ámbito laboral y de recreación. Permitiendo desarrollar sus destrezas, enfrentar el estrés diario, trabajar productivamente, las que se relacionan con la salud física y el bienestar social (MINSA, 2018). Se expresa a través del comportamiento y la interacción interpersonal (Organización Mundial de la Salud [OMS], 2013).

Al respecto Achenbach y Edelbrock (1987; en Ivanova, et al. 2007) clasifican las dificultades que presentan los adolescentes en dos dimensiones. Una de ellas, designada trastorno externalizante, compuesto por comportamientos que generan detrimento o incordio en el hábitat de la persona, tales como dificultades de conducta y comportamientos agresivos; la otra, trastorno internalizante, el que incluiría respuestas emocionales y cognitivas hacia el mismo individuo; las que se expresa como respuestas de ansiedad, depresión, problemas de relación y quejas somáticas. Buendía (1999) y otros investigadores han demostrado la utilidad de esta clasificación señalando que estas dimensiones problemas difiere según el sexo, siendo los hombres quienes tienen más propensión a exteriorizar dificultades externalizantes en relación con las mujeres, las que presentarían más complicaciones de tipo internalizante (Zubeidat, Fernández-Parra, Ortega, Vallejo y Sierra, 2009; Bartels, Cacioppo, van Beijsterveldt \& Boomsma, 2013; Rosenfield \& Mouzon, 2013).

Los hombres se expondrían a más peligros, tanto personales como del entorno, en la ingesta de sustancias (López Larrosa \& Rodríguez-Arias Palomo, 2010. Citados en Castañeda, 2014), presentarían más conductas delictivas (González y Cueto, 2000), mayor impulsividad y puntajes más altos en conductas antisociales (García y Orellana, 2009).

Las mujeres, en contraste, manifestarían altos niveles en ansiedad, quejas somáticas y depresión (González y Cueto, 2000); asimismo mostrarían mayor empatía y al mismo tiempo ellas tienden a vincularse con los demás y su entorno (García y Orellana, 2009).

Una variable sustancial para evaluar los factores protectores y de riesgo en la salud mental del adolescente es el apoyo social percibido, pues permite el manejo de los problemas de salud y el bienestar psicológico (Barra, 2012).

El apoyo social (Cobb, [1976], citado en Nava, Bezies y Vega, 2015) es un factor relevante con a través del cual las personas descubren o entienden que son queridos, considerados, y contenidos dentro de la familia, los pares, y otras personas importantes. Así el mantener relaciones sociales se constituyen en una adecuada percepción de apoyo social. Para nuestra sociedad las redes de apoyo son por excelencia la familia y los amigos, acudiendo muy poco a personas 
ajenas (Nava y Vega, 2008). El apoyo social suscita conductas saludables en el individuo, favorece la disminución de respuestas desadaptativas frente a situaciones estresantes (Cutrona, Hessling \& Suhr; 1997). En la práctica clínica es cotidiano observar esta influencia, Erhardt (2016) encuentra una relación inversa entre depresión y el apoyo social, lo cual es similar cuando existe menor apoyo de la familia, los pares y otros que son importantes para la persona, tanto en varones y en mujeres. El soporte social proporciona al individuo un sentido de estabilidad, de previsión y de control, que lo hace sentirse mejor y percibir de manera más positiva su entorno (Barrón y Sánchez, 2001; Musitu y Cava, 2002; Wight, Botticello y Aneshensel, 2006; citados en Barra, 2012). Los individuos satisfechos, asertivos, con habilidades sociales, buena valoración de sí mismos y comunicativos, tienden a distinguir mayores niveles de apoyo de distintas redes sociales y señalan sentir satisfacción consigo mismo (Acuña y Bruner, 1999; Aduna, 1998; Sarason, et al, 1990; citados en Rodriguez, 2011; y Gurung, Sarason, B. \& Sarason, I., 1997. En cambio, sujetos aprehensivos, con desánimo, baja autoestima, locus de control externo y falta de gusto con la vida habitualmente señalar mínimos grados de sostén social, ello puede perturbar de forma inapropiada la percepción de su aprecio a sí mismo (Daniels \& Guppy, 1997; Gurung, Sarason, B., Sarason, I.,1997).

Frente a ello se plantearon como objetivos para el estudio, analizar la relación entre las conductas problemas de la salud mental con las fuentes del apoyo social percibido; así también evidenciar las diferencias entre las conductas problemas de la salud mental con las fuentes del apoyo social percibido según género, en escolares de cuarto grado de secundaria de Instituciones Educativas Públicas de Lima Metropolitana.

\section{MÉTODO}

El tipo de estudio es sustantivo, método correlacional. El diseño es ex post facto, transversal, los datos fueron recogidos en un solo momento (Hernández, Fernández y Baptista, 2010).

\section{Muestra}

Se escogieron las Instituciones educativas tomando en cuenta la distribución geográfica que utilizan las Direcciones Regionales de Salud (DIRESA) del Ministerio de Salud; Lima ciudad, norte, sur y este, para el acopio de los datos, asimismo se obtuvo la población a partir de la data de Escale del Minedu. Se aplicó los instrumentos de forma grupal. Para la determinación del tamaño muestral se aplicó la fórmula para población finita. 
La muestra quedo compuesta por 627 escolares de cuarto grado de secundaria de Instituciones Educativas Públicas de Lima metropolitana; en cuanto al género masculino lo constituyeron $360(57,4 \%)$ y el femenino 267 (42,6\%). Respecto a la edad la mayor proporción de evaluados tienen 15 años (62.8\%), seguido de los de 16 años (22.3\%), en menor porcentaje se encuentran los de 18 años $(0.6 \%)$. En relación al estado civil de los padres, el $28.5 \%$ son convivientes, el $28.1 \%$ son casados, viven solo con uno de los padres un $22.8 \%$, y padres viudos el $3.0 \%$.

\section{Variables de Estudio}

Pertenecen a los indicadores del Inventario de Problemas conductuales, compuesta por dos dimensiones y ocho conductas problemas; Externalizante: Problemas sociales, de pensamiento, búsqueda de atención, de conducta y conducta agresiva; e Internalizante: conducta ansiosa, problemas de relación y somatización. Y las de la Escala multidimensional del apoyo social percibido de Zimet, Dahlem, Zimet \& Farley (1988) compuesta por tres fuentes, apoyo de la familia, de los pares y otros significativos.

\section{Técnicas e instrumentos de recolección de datos.}

Se empleó la técnica de la indagación y dos instrumentos, el Inventario de Problemas Conductuales (Achenbach, 1991) afinado por Majluf (1999, y la versión adaptada de la Escala multidimensional del apoyo social percibido de Zimet (MSPSS) (Zimet, Dahlem, Zimet \& Farley, 1988, adaptada por Cárdenas (2016) en Lima, y para establecer los datos socio-demográficos el investigador confeccionó una ficha de para recoger las edades, sexo y procedencia. Ambos instrumentos fueron adaptados y validados para la muestra de escolares de cuarto grado de secundaria.

\section{Instrumentos:}

Ficha de datos socio-demográficos: Elaborada por el investigador. Aplicada de manera agrupada. Permite recoger características relevantes de los sujetos estudiados como, edad, sexo, procedencia, con quién vive, estado civil de padres.

Inventario de Problemas Conductuales (Achenbach, 1991) adaptado en el Perú por Majluf (1999), compuesta por dos dimensiones, externalización con cinco conductas problemas (problemas sociales, de pensamiento, búsqueda de atención, de conducta y conducta agresiva) e internalización con tres (conducta ansiosa, problemas de relación y somatización). Majluf obtiene una fiabilidad test-retest de 0.92 . La validez del inventario la obtuvo a través de criterio clínico externo. Asimismo, Castañeda (2014) obtiene un Alpha de Cronbach de 0.93 en la escala total, y coeficientes apropiados para las escalas de externalización $(\alpha=0.86)$ e internalización $(\alpha=0.87)$ y para la validez discriminaciones ítem- 
test mayores a 0.30. En el actual estudio se alcanzó una consistencia interna Alpha de Cronbach 0.91 en la prueba total, en sus dimensiones, externalización e internalización, un Alpha de Cronbach de 0.86 y 0.848 respectivamente y su validez de construcción se realizó a través del método ítem-test con valores para toda la escala entre .241 y .472 ,

Escala multidimensional del apoyo social percibido elaborado por de Zimet (MSPSS) (Zimet, G., Dahlem, Zimet, S. \& Farley, 1988); consta de tres fuentes de apoyo, familia, amigos y otros significativo. En Lima Cárdenas (2016) consigue una confiabilidad alfa de Cronbrach de .87. La validez de contenido consigue un coeficiente de Aiken de $.95 \mathrm{y}$ en la validez usando el método ítem-test los valores oscilan entre .327 y .667. En el presente estudio se halló un Alpha de Cronbach de 0.853 en el cuestionario total, y la validez a través del método ítemtest con valores entre .448 y .620 al revisar sus tres fuentes, lo que es indicador que la prueba mide en relación con los márgenes de error permitido.

\section{RESULTADOS}

Para las correlaciones se utilizó análisis bivariado y multivariado.

1. Relación entre las conductas problemas de la salud mental con las fuentes del apoyo social percibido en escolares de cuarto grado de secundaria de Instituciones Educativas Públicas de Lima metropolitana.

Análisis bivariado: En la muestra total, existen correlaciones estadísticamente significativas $(\mathrm{p}<0.05)$ e inversas entre el apoyo social familiar con las conductas problema en general, así como en sus dimensiones externalizantes e internalizantes, específicamente con las conductas de problemas sociales, de pensamiento, búsqueda de atención, conducta agresiva, ansiosa y problemas de relación. Lo cual nos indica que los adolescentes que perciben mayor apoyo social de parte de su familia, tienden a presentar en menor medida estas conductas problemas, Tabla 1.

Análisis multivariado, busca estimar cuáles de las variables predictoras, llámese conductas problema, pueden explicar de forma significativa al apoyo social considerando todas las variables en conjunto. Encontrando se observan que las variables que resultan estadísticamente significativas $(\mathrm{p}<.05)$ son conducta ansiosa y problemas de relación. Luego tanto el signo del valor B como el Exp (B) nos indican que, en cuanto a las conductas problemas, los evaluados que tienden a presentar menor nivel de apoyo social son quienes presentan mayores conductas problema de tipo conducta ansiosa y problemas de relación. 
Salud mental y apoyo social percibido en escolares de cuarto grado de secundaria de Instituciones Educativas públicas de Lima Metropolitana

Tabla 1

Correlaciones entre conductas problemas y las dimensiones del apoyo social en la muestra total

\begin{tabular}{|c|c|c|c|c|}
\hline & & Apoyo social familiar & Apoyo social amigos & $\begin{array}{c}\text { Apoyo social persona } \\
\text { significativa }\end{array}$ \\
\hline \multirow{2}{*}{$\begin{array}{l}\text { Conductas problema } \\
\text { Total }\end{array}$} & Rho &,$- 255^{* * *}$ &,$- 103^{*}$ &,$- 143^{* *}$ \\
\hline & $\mathrm{p}$ & .000 & .010 & .000 \\
\hline \multirow[t]{2}{*}{ Externalización } & Rho &,$- 219^{* *}$ & -.062 &,$- 104^{* *}$ \\
\hline & $\mathrm{p}$ & .000 & .122 & .009 \\
\hline \multirow{2}{*}{ Problemas sociales } & Rho &,$- 245^{* *}$ &,$- 160^{* *}$ &,$- 214^{* *}$ \\
\hline & $\mathrm{p}$ & .000 & .000 & .000 \\
\hline \multirow[t]{2}{*}{ Problemas de pensamiento } & Rho &,$- 104^{* *}$ & -.064 & -.056 \\
\hline & $\mathrm{p}$ & .009 & .112 & .158 \\
\hline \multirow[t]{2}{*}{ Búsqueda de atención } & Rho &,$- 206^{* *}$ & -.047 &,$- 138^{* *}$ \\
\hline & $\mathrm{p}$ & .000 & .243 & .001 \\
\hline \multirow{2}{*}{ Problemas de conducta } & Rho & -.047 & .015 & .024 \\
\hline & $\mathrm{p}$ & .235 & .716 & .553 \\
\hline \multirow[t]{2}{*}{ Conducta agresiva } & Rho &,$- 235^{* *}$ & -.020 & -.069 \\
\hline & $\mathrm{p}$ & .000 & .615 & .086 \\
\hline \multirow[t]{2}{*}{ Internalización } & Rho &,$- 264^{* *}$ &,$- 146^{* *}$ &,$- 179^{* * *}$ \\
\hline & $\mathrm{p}$ & .000 & .000 & .000 \\
\hline \multirow[t]{2}{*}{ Conducta ansiosa } & Rho &,$- 318^{* *}$ &,$- 134^{* * *}$ &,$- 214^{* *}$ \\
\hline & $\mathrm{p}$ & .000 & .001 & .000 \\
\hline \multirow[t]{2}{*}{ Problemas de relación } & Rho &,$- 289^{* *}$ &,$- 174^{* *}$ &,$- 237^{* *}$ \\
\hline & $\mathrm{p}$ & .000 & .000 & .000 \\
\hline \multirow[t]{3}{*}{ Somatización } & Rho & -.066 & -.070 & -.002 \\
\hline & $\mathrm{p}$ & .097 & .079 & .951 \\
\hline & $\mathrm{N}$ & 627 & 627 & 627 \\
\hline
\end{tabular}

**. La correlación es significativa en el nivel 0,01 (bilateral).

*. La correlación es significativa en el nivel 0,05 (bilateral).

2. Relación entre las conductas problemas de la salud mental con las fuentes del apoyo social percibido en escolares de cuarto grado de secundaria de Instituciones Educativas Públicas, según sexo, de Lima metropolitana.

\section{Correlación Bivariada. Varones}

Existe correlaciones significativas $(p<0.05)$ e inversas entre el apoyo social familiar y apoyo social de parte de personas significativas con las conductas problema en general, y sus dos dimensiones externalizantes e internalizantes, en 
especial con las conductas de problemas sociales, búsqueda de atención, agresiva, ansiosa y problemas de relación.

Con respecto al apoyo social de los amigos, existen correlaciones estadísticamente significativas $(\mathrm{p}<0.05)$ e inversas con las conductas problema de la dimensión internalizante: conducta ansiosa y problemas de relación. Como se aprecia en la Tabla 2.

Tabla 2

Correlaciones entre conductas problemas y las dimensiones del apoyo social en los varones

\begin{tabular}{|c|c|c|c|c|}
\hline & & Apoyo social familiar & Apoyo social amigos & $\begin{array}{c}\text { Apoyo social persona } \\
\text { significativa }\end{array}$ \\
\hline \multirow{2}{*}{ Conductas problema } & Rho &,$- 155^{* *}$ & -.045 &,$- 115^{*}$ \\
\hline & $\mathrm{p}$ & .003 & .396 & .029 \\
\hline \multirow{2}{*}{ Externalización } & Rho &,$- 150^{* *}$ & -.016 & -.087 \\
\hline & $\mathrm{p}$ & .004 & .765 & .101 \\
\hline \multirow{2}{*}{ Problemas sociales } & Rho &,$- 163^{* *}$ & -.092 &,$- 148^{* *}$ \\
\hline & $\mathrm{p}$ & .002 & .082 & .005 \\
\hline \multirow{2}{*}{ Problemas de pensamiento } & Rho & -.069 & -.028 & -.092 \\
\hline & $\mathrm{p}$ & .194 & .596 & .083 \\
\hline \multirow{2}{*}{ Búsqueda de atención } & Rho &,$- 171^{* * *}$ & -.015 &,$- 129^{*}$ \\
\hline & $\mathrm{p}$ & .001 & .777 & .014 \\
\hline \multirow{2}{*}{ Problemas de conducta } & Rho & -.012 & .063 & .047 \\
\hline & $\mathrm{p}$ & .819 & .231 & .371 \\
\hline \multirow{2}{*}{ Conducta agresiva } & Rho &,$- 181^{* *}$ & -.040 & -.064 \\
\hline & $\mathrm{p}$ & .001 & .453 & .225 \\
\hline \multirow{2}{*}{ Internalización } & Rho &,$- 159^{* *}$ & -.097 &,$- 153^{* *}$ \\
\hline & $\mathrm{p}$ & .002 & .066 & .004 \\
\hline \multirow{2}{*}{ Conducta ansiosa } & Rho &,$- 206^{* *}$ &,$- 118^{*}$ &,$- 196^{* *}$ \\
\hline & $\mathrm{p}$ & .000 & .025 & .000 \\
\hline \multirow{2}{*}{ Problemas de relación } & Rho &,$- 197^{* *}$ &,$- 116^{*}$ &,$- 201^{* * *}$ \\
\hline & $\mathrm{p}$ & .000 & .028 & .000 \\
\hline \multirow{2}{*}{ Somatización } & Rho & -.018 & -.031 & .016 \\
\hline & $\mathrm{p}$ & .737 & .563 & .765 \\
\hline
\end{tabular}

\section{Análisis Multivariado varones.}

En el caso de los varones, el valor p de la prueba de ómnibus para el modelo de los problemas de conducta alcanza un nivel de significación de 0.003 , el cual es menor a 0.05 , resultando significativo. Es decir, las variables seleccionadas para el análisis (conductas problema) sí pueden explicar el apoyo social para los varones. Se observó que las variables que resultan estadísticamente significativas $(\mathrm{p}<.05)$ 
son conducta ansiosa y problemas de relación. Luego tanto el signo del valor B como el Exp (B) nos indican que, en cuanto a los problemas de conducta, los varones que tienden a presentar menor nivel de apoyo social son quienes presentan mayores conductas problema de tipo conducta ansiosa y problemas de relación.

\section{Análisis Bivariado: Mujeres}

Existen correlaciones entre las conductas problemas con las dimensiones del apoyo social para las mujeres. Se evidencian correlaciones significativas $(p<0.05)$ e inversas entre el apoyo social familiar, apoyo social de parte de los amigos y apoyo social de parte de personas significativas con las conductas problema en general, y en sus dos dimensiones externalizantes e internalizantes, particularmente con las conductas problemas sociales, de pensamiento, búsqueda de atención, agresiva, ansiosa, de relación y somatización. Tal como se aprecia en la Tabla 3.

Tabla 3

Correlaciones entre conductas problemas y las dimensiones del apoyo social en las mujeres

\begin{tabular}{|c|c|c|c|c|}
\hline & & Apoyo social familiar & Apoyo social amigos & $\begin{array}{c}\text { Apoyo social persona } \\
\text { significativa }\end{array}$ \\
\hline \multirow{2}{*}{ Conductas problema } & Rho &,$- 354^{* *}$ &,$- 241^{\text {** }}$ &,$- 167^{* *}$ \\
\hline & $\mathrm{p}$ & .000 & .000 & .006 \\
\hline \multirow{2}{*}{ Externalización } & Rho &,$- 296^{\text {k* }}$ &,$- 166^{\text {*k }}$ &,$- 124^{*}$ \\
\hline & $\mathrm{p}$ & .000 & .006 & .043 \\
\hline \multirow{2}{*}{ Problemas sociales } & Rho &,$- 321^{* *}$ &,$- 279^{* *}$ &,$- 294^{* *}$ \\
\hline & $\mathrm{p}$ & .000 & .000 & .000 \\
\hline \multirow{2}{*}{ Problemas de pensamiento } & Rho &,$- 122^{*}$ &,$- 149^{*}$ & -.007 \\
\hline & $\mathrm{p}$ & .046 & .014 & .910 \\
\hline \multirow{2}{*}{ Búsqueda de atención } & Rho &,$- 232^{* *}$ &,$- 147^{*}$ &,$- 147^{*}$ \\
\hline & $\mathrm{p}$ & .000 & .016 & .016 \\
\hline \multirow{2}{*}{ Problemas de conducta } & Rho & -.091 & -.063 & -.006 \\
\hline & $\mathrm{p}$ & .136 & .308 & .919 \\
\hline \multirow{2}{*}{ Conducta agresiva } & Rho &,$- 305^{* *}$ & -.005 & -.070 \\
\hline & $\mathrm{p}$ & .000 & .931 & .257 \\
\hline \multirow{2}{*}{ Internalización } & Rho &,$- \mathbf{3 5 3}^{* k}$ &,$- 289^{* *}$ &,$- 205^{* *}$ \\
\hline & $\mathrm{p}$ & .000 & .000 & .001 \\
\hline \multirow{2}{*}{ Conducta ansiosa } & Rho &,$- 420^{* * k}$ &,$- 240^{* *}$ &,$- 235^{* k}$ \\
\hline & $\mathrm{p}$ & .000 & .000 & .000 \\
\hline \multirow{2}{*}{ Problemas de relación } & Rho &,$- 378^{* *}$ &,$- 292^{* * k}$ &,$- 279^{* *}$ \\
\hline & $\mathrm{p}$ & .000 & .000 & .000 \\
\hline \multirow{2}{*}{ Somatización } & Rho & -.096 &,$- 161^{* * *}$ & -.027 \\
\hline & $\mathrm{p}$ & .118 & .008 & .657 \\
\hline
\end{tabular}

**. La correlación es significativa en el nivel 0,01 (bilateral).

*. La correlación es significativa en el nivel 0,05 (bilateral). 


\section{Análisis multivariado mujeres.}

En el caso de las mujeres, el valor p de la prueba de ómnibus para el modelo de los problemas de conducta alcanza un nivel de significación de 0.000 , el cual es menor a 0.05 , resultando significativo. Es decir, las variables elegidas para el análisis (conductas problema) sí pueden explicar el apoyo social para las mujeres. Las variables que resultan estadísticamente significativas $(\mathrm{p}<.05)$ son problemas sociales y problemas de relación. Luego tanto el signo del valor B como el Exp (B) nos indican que, en cuanto a los problemas de conducta, las mujeres que tienden a presentar menor nivel de apoyo social son quienes presentan mayores conductas problema de tipo problemas sociales y problemas de relación.

3. Conocer las conductas problemas de la salud mental en los escolares de cuarto grado de secundaria de Instituciones Educativas Públicas de Lima metropolitana.

En la Tabla 4 se muestran la proporción de la presencia de las conductas problema en la muestra analizada. Es así que un 20.7\% presenta conductas problema a nivel general. En cuanto a las conductas de externalización, la que obtiene mayor porcentaje son los problemas de conducta con un $25.2 \%$. En cuanto a la internalización es la somatización la que alcanza mayor porcentaje, con un $23.1 \%$.

Tabla 4

Presencia de conductas problema

\begin{tabular}{lcccc}
\hline \multirow{2}{*}{ Conducta problema } & \multicolumn{2}{c}{ Ausencia } & \multicolumn{2}{c}{ Presencia } \\
\cline { 2 - 5 } & Fr & $\mathbf{\%}$ & Fr & \% \\
\hline Conductas problema Total & 497 & 79,3 & 130 & $\mathbf{2 0 , 7}$ \\
Externalización & 489 & 78,0 & 138 & 22,0 \\
Problemas sociales & 492 & 78,5 & 135 & 21,5 \\
Problemas de pensamiento & 523 & 83,4 & 104 & 16,6 \\
Búsqueda de atención & 532 & 84,8 & 95 & 15,2 \\
Problemas de conducta & 469 & 74,8 & 158 & $\mathbf{2 5 , 2}$ \\
Conducta agresiva & 502 & 80,1 & 125 & 19,9 \\
Internalización & 485 & 77,4 & 142 & 22,6 \\
Conducta ansiosa & 487 & 77,7 & 140 & 22,3 \\
Problemas de relación & 504 & 80,4 & 123 & 19,6 \\
Somatización & 482 & 76,9 & 145 & $\mathbf{2 3 , 1}$ \\
\hline
\end{tabular}

4. Conocer las fuentes del apoyo social percibido en los escolares de cuarto grado de secundaria de Instituciones Educativas Públicas de Lima metropolitana.

Apoyo social total la mayoría de los evaluados se ubica en el nivel medio, siendo el $56.0 \%$, le siguen quienes se encuentran en el nivel alto con un $31.3 \%$ 
y finalmente la menor proporción se ubica en el nivel bajo, representando el $12.8 \%$. Similar proporción se cumple en todas las dimensiones del apoyo social.

5. Comparar las conductas problemas de la salud mental en los escolares de cuarto grado de secundaria de Instituciones Educativas Públicas, según sexo, de Lima metropolitana.

Existen diferencias estadísticamente significativas $(\mathrm{p}<0.05)$ en las conductas problema de tipo sociales, de pensamiento, búsqueda de atención, conducta ansiosa, problemas de relación y somatización entre hombres y mujeres. Es así que según los valores de los rangos promedio, son las mujeres las que presentan estos tipos de conducta problema en mayor medida que los varones, además las mujeres presentan mayor diferencia de medias en problemas de conducta y en conducta agresiva. Como se aprecia en la Tabla 5.

Tabla 5

Comparación de las conductas problema según sexo

\begin{tabular}{lllll}
\hline Conducta problema & U de Mann-Whitney & \multicolumn{1}{c}{$\begin{array}{c}\text { Rango medio para } \\
\text { hombres } \\
(\mathbf{N}=\mathbf{3 6 0})\end{array}$} & $\begin{array}{c}\text { Rango medio para } \\
\text { mujeres } \\
(\mathbf{N}=\mathbf{2 6 7})\end{array}$ & p \\
\hline Sociales & 41012.000 & 294.42 & $\mathbf{3 4 0 . 4 0}$ & $\mathbf{. 0 0 2}$ \\
De pensamiento & 40290.500 & 292.42 & $\mathbf{3 4 3 . 1 0}$ & $\mathbf{. 0 0 1}$ \\
Búsqueda de atención & 40115.000 & 291.93 & $\mathbf{3 4 3 . 7 6}$ & $\mathbf{. 0 0 0}$ \\
De conducta & 45912.000 & 308.03 & 322.04 & .335 \\
Conducta agresiva & 45365.500 & 306.52 & 324.09 & .228 \\
Conducta ansiosa & 31960.000 & 269.28 & $\mathbf{3 7 4 . 3 0}$ & $\mathbf{. 0 0 0}$ \\
De relación & 39175.000 & 289.32 & $\mathbf{3 4 7 . 2 8}$ & $\mathbf{. 0 0 0}$ \\
Somatización & 39640.500 & 290.61 & $\mathbf{3 4 5 . 5 3}$ & $\mathbf{. 0 0 0}$ \\
\hline
\end{tabular}

6. Comparar las fuentes del apoyo social percibido en los escolares de cuarto grado de secundaria, según sexo, de Instituciones Educativas Públicas de Lima metropolitana.

En la tabla 6 se observa según los valores $\mathrm{p}$, que existe diferencias significativas $(p<0.05)$ en las dimensiones apoyo social familiar y de amigos. Es así que según los valores de los rangos promedio son los varones (333.66) los que distinguen mayor apoyo social de la familia en comparación con las mujeres (287.50). Por el contrario, son las mujeres (335.81) quienes perciben mayor apoyo social de los compañeros, en contraste con los varones (297.83). 
Tabla 6

Comparación del apoyo social y sus dimensiones según sexo

\begin{tabular}{lllll}
\hline \multicolumn{1}{c}{ Apoyo Social } & U de Mann-Whitney & $\begin{array}{c}\text { Rango medio para } \\
\text { hombres } \\
(\mathbf{N = 3 6 0 )}\end{array}$ & $\begin{array}{c}\text { Rango medio para } \\
\text { mujeres } \\
\mathbf{( N = \mathbf { 2 6 7 } )}\end{array}$ & p \\
\hline Apoyo social total & 47161.000 & 316.50 & 310.63 & .688 \\
Apoyo social familiar & 40983.500 & $\mathbf{3 3 3 . 6 6}$ & 287.50 & $\mathbf{. 0 0 2}$ \\
Apoyo social amigos & 42238.000 & 297.83 & $\mathbf{3 3 5 . 8 1}$ & $\mathbf{. 0 0 9}$ \\
Apoyo social persona significativa & 47650.000 & 315.14 & 312.46 & .854 \\
\hline
\end{tabular}

\section{Interpretación de los datos o discusión}

Al constituir los adolescentes peruanos de 10 a 19 el 18.6\% (INEI; 2015) de la población, ello resulta preocupante cuando se revisan las estadísticas respecto a su salud en general y en especial a su salud mental como lo reporta el Ministerio de Salud (2017), pues se encuentra conducta violenta, acto delictivo, planeación suicida, depresión estas dos últimos con mayor predominancia en Lima y Callao, también ansiedad generalizada, problemas alimentarios. Asimismo, el INEI (2015) con respecto a la presencia de violencia, el porcentaje de adolescentes víctimas de violencia física o psicológica, alguna vez en la vida, en sus hogares o centros de atención residencial fue de $81.0 \%$, mientras que en la institución educativa era de $73.8 \%$. Estos datos nos permiten considerar la relevancia de estudiar las conductas problemas en relación al apoyo social percibido. Muchos de estos problemas de conducta y trastornos emocionales, como lo reporta el Ministerio de Salud (2017) se encuentran asociados a desavenencias familiares y, distintas formas de maltrato. Ello nos confirma apreciar el papel que tiene la familia, los pares y otras personas significativas. Datos que corroboramos en el presente estudio al hallar relación inversa entre el apoyo social percibido hacia la familia, los amigos y otras personas significativas con las conductas problemas, es decir que los examinados que perciben mayor nivel de apoyo social son quienes presentan menores conductas problemas; tanto internalizantes como externalizantes; datos que armonizan con Gurung, Sarason, \& Sarason (1997) quienes señalan que individuos ansiosos, con humor depresivo, autoestima disminuida, locus de control externo suelen percibir rangos bajos de apoyo social, afectando su autovaloración. Lambert, Lalongo, Boyd, \& Cooley (2005) manifiestan asociación entre los síntomas depresivos y ansiosos como reguladores de la asociación que se presenta entre la conducta agresiva y el estar expuesto a violencia en la comunidad resaltando la relación parental y el grupo de pares.

Según género, tanto varones como mujeres, en la correlación bivariada, es inversa, entre el soporte social familiar con las conductas problema en general $\mathrm{y}$ en sus dos dimensiones. El papel de la familia es un factor protector; lo cual concuerda con lo propuesto por Nava y Vega (2008) quienes consideran que en 
la sociedad las redes de apoyo son por excelencia la familia y amistades; además Huerta et al. (2015) observan en alumnos de secundaria que la violencia entre los padres coadyuva en la generación de problemas de depresión en las mujeres y conductas agresivas en los varones. Las características de la relación familiar son definitorias en la presentación de problemas de salud mental (OMS, 2011).

Al analizar las conductas problema de los escolares del estudio, estas se hallan por debajo del punto de corte como para asumir de manera importante la presencia de una de ellas. Es así que un 20,7\% presentan conductas problema a nivel general, en cuanto a los problemas de externalización se aprecian los problemas de conducta y sociales y, en internalización destacan la somatización y la conducta ansiosa. Fowler, Tompsett y Baltes (2009, citados en Cárdenas, 2016) indican que en los adolescentes existe una relación potente entre estar expuesto a violencia con dificultades de externalización, lo que puede conllevar a que los problemas de conducta, que se han encontrado y sociales, se hallen vinculados con este hallazgo, afectando su salud mental lo que puede conllevar a que se involucren en conductas de riesgo.

Huerta y cols $(2015,2018)$, evidencian que la violencia dentro de la familia incrementa la presencia de problemas internalizantes, observando la depresión en ambos sexos y externalizantes, en el incremento de la respuesta agresiva acarreando el descuido en su salud y menoscabo en sus razones para vivir; afirmación sustentada por otros estudios (Fagan, 2003; Hollins, 2008; Li et al.,2002) que remarcan que la violencia coadyuva en la manifestación de señales de dificultades internalizantes, tales como ansiedad, depresión y estrés postraumático. Lo cual hace necesario tomarlo muy en cuenta en su prevención e intervención.

De acuerdo a lo obtenido son las mujeres quienes tienden a presentar más conductas problemas en comparación con los hombres (problemas sociales, búsqueda de atención, ansiedad, de relación y somatización).

Resultados preocupantes pues los estudios de Zubeidat et al. (2009), Bartels et al. (2013) y Rosenfield \& Mouzon, (2013) indican que son los adolescentes varones quienes tienden a exhibir dificultades externalizantes al ser comparados con las mujeres, quienes revelarían más dificultades de tipo internalizante; datos que en el presente estudio se aprecian que los problemas de las dos dimensiones, mayormente ocurren en las mujeres, conductas problemas que afectarán su salud mental y la ponen en mayor riesgo. En el mismo sentido González y Cueto, (2000) indican menor protección y mayor riesgo en los varones para consumir sustancias psicoactivas y conductas criminales $\mathrm{y}$, en las mujeres, encuentran indicadores altos en depresión, ansiedad y quejas somáticas.

Resultados que refuerzan la situación de riesgo en que se encuentran las mujeres afectando los diferentes aspectos de su vida cotidiana. 
Por otro lado, Garaigordobil, Durá y Pérez (2005) encuentran que la autoestima y el autoconcepto inciden en la menor presentación de conductas problema, menor patología y por ende mejor salud mental; en conexión a ello Castañeda (2014) señala que las adolescentes presentan menor autoestima y que sus conductas problema internalizante son mayores que en los hombres quienes tienden a tener una mejor percepción de su autoconcepto en contraste con las mujeres, las cuales se vinculan con la presencia de problemas externalizantes en ambos.

Ello nos permite afirmar la existencia de otras variables que inciden en la salud mental de los adolescentes que ameritan seguir estudiando, una de estas variables es la violencia entre los padres y/o los conflictos parentales pues son las mujeres, de acuerdo a Peltonen, Ellonen, Larsen y Helweg-Larsen, (2010, citados en Salas, 2017) quienes están más expuestas en contraste con los hombres, agregan que ellas no solo soportan mayor violencia familiar, sino también fuera de casa. Huerta, et al (2018) evidencian en el sexo femenino que las creencias para vivir, se hacen incuestionables cuando no existe violencia en la interacción familiar, lo que permite destacar la fuerte influencia en la salud mental, a la familia.

Los varones tienen mayor percepción de apoyo de parte de la familia y las mujeres de parte de los amigos. Salas, (2017) señala que el no tener a alguien que sea significativo para el adolescente aumenta la posibilidad de exponerse a situaciones de riesgo. Una percepción apropiada del apoyo social indica Gurung, Sarason, B. y Sarason I. (1997) va a incidir en incrementar los sentimientos de seguridad y de bienestar personal, así como demasiado estrés y ansiedad perturban las habilidades sociales.

\section{CONCLUSIONES}

- La muestra total de escolares evidencia que hay correlación significativa e inversa entre las conductas problema en sus dimensiones externalizante e internalizante con las fuentes del apoyo social percibido. El menor apoyo social propicia mayor presencia de conductas problema ansiosa y problemas de relación, incidiendo en la salud mental del adolescente.

- Según género existe relación significativa e inversa tanto en hombres como en mujeres en problemas internalizantes como extrernalizantes.

- En varones se halla relación en el apoyo familiar y otras personas significativas con ambas dimensiones y en el caso de la percepción del soporte social con los amigos en la internalizante. En las mujeres es similar agregándose la asociación inversa con las dos dimensiones y las tres fuentes de apoyo social en las conductas problemas sociales.

- El 20,7\% de la muestra presenta conductas problema a nivel general, en la dimensión externalizante obtiene mayor porcentaje las dificultades 
de conducta (25.2\%) seguido de los problemas sociales (21.5\%); en internalizante están somatización (23.1\%) y conducta ansiosa (22.3\%).

- La percepción del apoyo social es apropiada en la muestra total; el mayor porcentaje de adolescentes exhiben un nivel medio (56,0\%), nivel alto $31,3 \%$ y bajo $12,8 \%$, de la misma manera se aprecia en sus tres fuentes.

- Son las mujeres quienes presentan conductas problemas de manera significativa, en comparación con los varones, tales como problemas sociales, de pensamiento, búsqueda de atención, conducta ansiosa, de relación y somatización. Lo cual afecta la salud mental de la mujer e incrementa su situación de riesgo en esta etapa de vida.

- Los varones perciben mayor apoyo social familiar que las mujeres, por otro lado, las mujeres distinguen mayor soporte social de las amistades en contraste con los hombres.

\section{REFERENCIAS}

Achenbach, T. (1991). Manual for the Youth Self Report and profile. Burlington VT: University of Vermont.

Barra, E. (2012). Influencia de la autoestima y del apoyo social percibido sobre el bienestar psicológico de estudiantes universitarios chilenos. Diversitas: Perspectivas en Psicología, 8(1), 29-38. Recuperado de http://www.redalyc.org/articulo. oa?id $=67923973002$

Bartels, M., Cacioppo, J., van Beijsterveldt, T. \&Boomsma, D. (2013). Exploring the Association between Well-Being and Psychopathology in Adolescents. 43(3), 177190. doi: 10.1007/s10519-013-9589-7

Buendía, J. (1999). Familia y Psicología de la Salud. Madrid, España: Pirámide.

Cárdenas, M. (2016). Funcionamiento familiar, soporte social percibido y autoeficacia para el afrontamiento del estrés como factores asociados al bienestar psicológico en estudiantes de una universidad privada de Trujillo-La Libertad. Revista de Psicología, 18(1), 72-85. doi: 10.18050/revpsi.v18n1a6.2016

Castañeda, K. (2014). Autoestima, Claridad de Autoconcepto y Salud Mental en adolescentes de Lima Metropolitana Tesis para optar el título de Licenciada en Psicología. PUCP. Recuperado en tesis.pucp.edu.pe/.../CASTANEDA_DIAZ_ ANNA_AUTOESTIMA_ADOLESCENTE...

Cutrona, C., Hessling, R. \& Suhr, J. (1997). The influence of husband and wife personality on marital social support interactions. Personal Relationships, 4, 379-393.

Daniels, K. \& Guppy, A. (1997). Stressors, locus of control and social support as consequences of afective psychological wellbeing. Journal of Occupational Health Psychology, 2(2), 156- 174. 
Defensoría del Pueblo (2008). Salud Mental y Derechos Humanos Supervisión de la política pública, la calidad de los servicios y la atención a poblaciones vulnerables. Primera Edición: Lima, Perú. Recuperado de http://www.defensoria.gob.pe

De Irala, J., Corcuera, P., Osorio, A. y Rivera, R. (2010). Estilos de vida de los adolescentes peruanos. Lima: Universidad de Piura, Universidad de Navarra, Intermedia Consulting. Recuperado de http://www.slideshare.net/intermediaconsulting/libroestilo-de-vida-de-los-adolescentes-peruanos

Erhardt, E. (2016). Relación entre apoyo social percibido en jóvenes de 18 a 30 años originarios del interior del país que asisten a la universidad en Capital Federal y el riesgo de desarrollar depresión. Tesis Licenciatura en Psicología. Argentina. Recuperado en https://repositorio.uade.edu.ar/xmlui/bitstream/handle/123456789/.../ Erhardt.pdf?...1

Fagan, A. (2003). The Short- and Long-Term Effects of Adolescent Violent Victimization Experienced Within the Family and Community. Violence and Victims, 18(4), 445 459. doi: 10.1891/vivi.2003.18.4.445

García, J., \& Orellana, M. D. C. (2009). Variables Psicológicas Moduladoras de la Autodefinición del Perfil en Procesos de Acoso Escolar: El papel del género y el curriculum escolar. European Journal of Education and Psychology, 1(2), 41-55.

Garaigordobil, M., Durá, A. \& Pérez, J.I. (2005). Síntomas psicopatológicos, problemas deconducta y autoconcepto-autoestima: Un estudio con adolescentes de 14 a 17 años. Anuario de Psicología Clínica y de la Salud (1) ,53-63.

González, L. y Cueto, E. (2000). El rendimiento escolar y los trastornos emocionales y comportamentales. Psicothema, 12, 340-343.

Gurung, R. A., Sarason, B. \& Sarason, I. (1997). Personal characteristics, relationship quality and social support perceptions and behavior in young adult romantic relationship. Personal Relationship, 4, 319-339.

Hernández, R.; Fernández, C. y Baptista, P. (2010). Metodología de la Investigación. Bogota, Colombia: Editorial Mc Graw Hill.

Hollins, L.D. (2008). Influence of exposure to child maltreatment, domestic violence and community violence on youth. Dissertation Abstracts International: Section B: The Sciences and Engineering, 69 (6-B), 3874.

Huerta, R.; Aliaga, J.; Santivañez, R.; Ramírez, N.; Ramos, J., Murillo, L., \& ...Pérez, P. (2015) Depresión y cólera-hostilidad en alumnos víctimas de violencia intrafamiliar, de cuarto grado de secundaria de instituciones educativas estatales de zonas urbano marginal de la ciudad de Lima. Revista de Investigación en Psicología. Facultad de Psicología. UNMSM. 18(2), 83-100.

Huerta, R.; Santivañez, R.; Ramirez, N.; Delgado, E.; Ramos, J.; y Oré, B. (2018) Razones para vivir y conducta de salud en estudiantes universitarios según procedencia de la ciudad de Lima. Revista de Investigación en Psicología. Facultad de Psicología. UNMSM. 21(2), 147-168. http://revistasinvestigacion.unmsm.edu.pe/index.php/psico 
Instituto Nacional de Estadística e Informática (2010). Nota de prensa. Recuperado de http://www.inei.gob.pe/

Instituto Nacional de Salud Mental Honorio Delgado - Hideyo Noguchi. (2012). Nota de Prensa 12/11.Lima-Perú. Recuperado en www.insm.gob.pe/oficinas/comunicaciones/ notasdeprensa/2012/011.html

Instituto Nacional de Estadística e Informática (INEI, 2015). Perú: Compendio Estadístico 2015. Lima, Perú.

Ivanova, M. Y., Achenbach, T. M., Rescorla, L. A., Dumenci, L., Almqvist, F., 29 Bilenberg,N., \& ... Verhulst, F. C. (2007). The generalizability of the Youth SelfReport syndrome structure in 23 societies.Journal of Consulting and Clinical Psychology, 75(5), 729-738. doi:10.1037/0022-006X.75.5.729

Lambert, S., Lalongo, N., Boyd, R. \& Cooley, M. (2005). Risk Factors for Community Violence Exposure in Adolescence. American Journal of Community Psychology, 36(1-2), 29-48. doi: 10.1007/s10464-005-6231-8

Li, X., Stanton, B., Pack, R., Harris, C., Cottrell, L. \& Burns, J. (2002). Risk and protective factors associated with gang involvement among urban African American adolescents. Youth \& Society, 34(2), 172-194. doi: 10.1177/004411802237862

Majluf, A. (1999) Psicopatología en adolescentes de Lima según el inventario de problemas conductuales de Achenbach. Revista de psicología de la Pontificia Universidad Católica del Perú, 17(01), 47-71.

Ministerio de Salud (2017). Situación de Salud de los Adolescentes y Jóvenes en el Perú. 2017 / -1a ed.- Ministerio de Salud - Lima-Perú. Recuperado de bvs.minsa.gob.pe/ local/MINSA/4143.pdf

Ministerio de Salud (2018). Documento Técnico. Plan nacional de fortalecimiento de Salud Mental Comunitaria 2018-2021. Ministerio de Salud. Lima-Perú.

Nava, C.; Bezies, R. y Vega, C. (2015) Adaptación y Validación de la Escala de Percepción de Apoyo Social de Vaux. LIBERABIT: Lima (Perú) 21(1): 49-58.

Nava, C. y Vega, V. (2008). Dinámica de red social y alteración psicológica en adolescentes con ausencia de familia de origen. Revista Diversitas - Perspectivas en Psicología, 4(2), 417-425. Recuperado de http://www.redalyc.org/pdf/679/67940214.pdf

Organización Mundial de la Salud (2011). El estado de salud de los adolescentes. Recuperado http://www.who.int/features/factfiles/adolescent_health/facts/es/index. html

Organización Mundial de la Salud (2013). Plan Nacional de salud mental 2013-2020. Recuperado de www.who.int/mental_health/publications/action_plan/es/. Ginebra, Suiza.

Rodríguez, S. (2011). Adaptación a niños del cuestionario MOS de apoyo social percibido. LIBERABIT: 17(2): 117- 128. Lima- Perú 
Rosenfield, S. \& Mouzon, D. (2013). Gender and Mental Health. Handbooks of Sociology and Social Research, 277-296. Springer Netherlands.doi: 10.1007/978-94-007-4276$5 \_14$

Salas. P. (2017). Exposición a la violencia, volición y salud mental en adolescentes institucionalizados. Tesis para optar al título de Licenciada en Psicología. Recuperado en tesis.pucp.edu.pe/repositorio/handle/123456789/972

Zimet, G.; Dahlem, N.; Zimet, S. \& Farley, G. (1988) The Multidimensional Scale of Perceived Social Support, J Pers Assess. 52, 30-41

Zubeidat, I., Fernández-Parra, A., Ortega, J.M., Vallejo, M.Á., \& Sierra, J.C. (2009). Características psicosociales y psicopatológicas en una muestra de adolescentes españoles a partir del YouthSelf-Report/11-18. Anales de Psicología, 25(1) ,60- 69. 